\title{
Cryptocurrencies for the Payment of Products or Services: Risks, Accounting Practices and Regulations
}

\author{
David Allen ${ }^{1}$, James Aselta ${ }^{1} \&$ Russell Engel ${ }^{1}$ \\ ${ }^{1}$ Department of Accounting, School of Business, Southern Connecticut State University, USA \\ Correspondence: Russell Engel, Department of Accounting, Southern Connecticut State University, New Haven, CT \\ 06515, USA. E-mail: engelr2@ southernct.edu
}

Received: August 16, 2019

Accepted: September 7, 2019

Online Published: September 9, 2019

doi:10.5430/afr.v8n4p19

URL: https://doi.org/10.5430/afr.v8n4p19

\begin{abstract}
This paper examines the risks, accounting practices and disclosures of companies who accept cryptocurrency for the payment of products or services. We provide a brief history of cryptocurrency and blockchain technology that allows the reader to deepen their understanding of the subject before moving on to a discussion of how regulatory bodies such as the Financial Accounting Standards Board (FASB), the Securities and Exchange Commission (SEC) and the Internal Revenue Service (IRS) are treating the accounting for cryptocurrency transactions.
\end{abstract}

Keywords: cryptocurrency, blockchain, tokens, reporting, disclosure

\section{Introduction}

Cryptocurrency is defined as "any form of currency that only exists digitally, that usually has no central issuing or regulating authority but instead uses a decentralized system to record transactions and manage the issuance of new units, and that relies on cryptography to prevent counterfeiting and fraudulent transactions" (Merriam-Webster, 2018). The first cryptocurrency, Bitcoin, was created in 2008. It is not known who created Bitcoin. Satashi Nakamoto is the name used by the author of the whitepaper that introduced Bitcoin to the world. The paper, Bitcoin: A Peer-to-Peer Electronic Cash System (Nakamoto, 2008) describes the utility, desirability and functionality of Bitcoin. Nakamoto describes Bitcoin as a peer payment system where people can transact with each other without going through a third-party vendor (like one must do with Paypal or Venmo). The workaround Nakamota proposes is a system called proof-of-work "We propose a solution to the double- spending problem using a peer-to-peer network. The network timestamps transactions by hashing them into an ongoing chain of hash-based proof-of-work, forming a record that cannot be changed without redoing the proof-of-work." The reward for doing proof-of-work is Bitcoin. This is known as Bitcoin Mining. The proof-of-work chain is more commonly known as the blockchain.

A blockchain or distributed ledger technology (DLT) is a "digitized, decentralized, public ledger of all cryptocurrency transactions. Constantly growing as 'completed' blocks (the most recent transactions) are recorded and added to it in chronological order" (Fortney, 2019).

Vitalik Buterin, the founder of the second most valuable cryptocurrency Ethereum, describes the benefits of cryptocurrency and the blockchain as reducing costs, removing points of failure, preventing censorship and ensuring transparency and trust (Buterin, 2014).

Early adopters of Bitcoin treated the cryptocurrency as a novelty. There are many stories of people having thousands of Bitcoins when they were nearly worthless and using them simply for the fun of using a digital form of money to buy physical goods. Bitcoin then gained a reputation as a currency to be used for illicit activities. Marketplaces for illegal goods and services, most notably the Silk Road, appeared online and Bitcoin became the preferable way to handle transactions on this platform. As Bitcoin was used more it became more stable, though still extremely volatile, and valuable. Bitcoin's increase in value inspired the creation of other cryptocurrencies and also grew large enough to draw the attention of investors and regulating bodies.

\section{Historical Trends}

In a clear violation of the stable dollar assumption, that a currency will remain stable over time, Bitcoin, Ethereum, and cryptocurrencies generally are extremely volatile. The total market for cryptocurrencies (historical and real time) can be viewed on coinmarketcap.com. Coinmarketcap.com lists over 1800 cryptocurrencies with a market cap of 
approximately \$177 Billion. The two Cryptocurrencies discussed earlier, Bitcoin and Ethereum, make up over $60 \%$ of the market. Bitcoin alone makes up over $50 \%$ of the total market. Table 1 shows the historical prices of Bitcoin and Ethereum. This table misses Bitcoin's December 2017 peak of \$20,089.

Table 1. Historical Prices of Bitcoin and Ethereum

\begin{tabular}{ccc}
\hline & $\begin{array}{c}\text { Dollar value of 1 } \\
\text { Bitcoin }\end{array}$ & $\begin{array}{c}\text { Dollar Value of 1 } \\
\text { Ethereum }\end{array}$ \\
\hline July 2019 & $\$ 11333$ & $\$ 268$ \\
July 2018 & $\$ 6643$ & $\$ 452$ \\
July 2017 & $\$ 2507$ & $\$ 305$ \\
July 2016 & $\$ 675$ & $\$ 12$ \\
July 2015 & $\$ 259$ & \\
July 2014 & $\$ 626$ & \\
July 2013 & $\$ 98$ & \\
\hline
\end{tabular}

Description: Historical prices of Bitcoin and Ethereum in US Dollars.

Table 2 presents the growth of the terms "cryptocurrency" and "Bitcoin" in the SEC Edgar database. This search can be replicated at https://searchwww.sec.gov/EDGARFSClient/jsp/EDGAR_MainAccess.jsp.

Table 2. Search Result of the Terms Cryptocurrency and Bitcoin from the SEC Edgar Database

\begin{tabular}{ccc}
\hline & $\begin{array}{c}\text { Mentions of } \\
\text { Cryptocurrency }\end{array}$ & Mentions of Bitcoin \\
\hline $\begin{array}{c}\text { Through July 2019 } \\
2018\end{array}$ & 1258 & 806 \\
2017 & 294 & 1626 \\
2016 & 79 & 486 \\
2015 & 60 & 272 \\
& & 592 \\
\hline
\end{tabular}

\section{Description: Search Result of the Terms Crypt
3. Companies Accepting Cryptocurrency}

The most widely accepted cryptocurrency is Bitcoin. The website 99 Bitcoins provides a list of over 50 companies that accept Bitcoin. This list includes fortune 500 companies like Microsoft to a small café, Coupa Café, in Palo Alto, California. Major companies that accept Bitcoin include Overstock, Expedia, Subway, PayPal, and Shopify (Sloan, 2018). It is important to consider what it means to "accept" cryptocurrency. Overstock appears to be a leader and is out on an island in this space. Overstock accepts and holds multiple cryptocurrencies whereas other companies "accept" Bitcoin by having a third-party process and convert the cryptocurrency into cash at the point of sale; a third party provides a guaranteed exchange rate. Two of the largest third-party Bitcoin payment processors are Bitpay and Coinbase. The protection they offer companies that accept cryptocurrency can be seen in their terms of service:

\subsection{Bitpay}

"We guarantee the exchange rate to you as long as the Purchaser pays within the proper time window after the invoice is created. Invoice timeout information is clearly displayed on each BitPay invoice (Bitpay, 2018).

\subsection{Coinbase:}

"The payment window has a short expiration time frame, when a customer pays for an item via Coinbase Commerce, there is a 15-minute window until the payment expires. If no payment has been made within that window, the order will expire and a new one will need to be created" (Coinbase, 2018). 


\section{Accounting for Cryptocurrency Transactions}

As discussed above, many corporations state that they accept cryptocurrency, but they do not, in fact, take ownership of cryptocurrency. They convert the cryptocurrency to their desired legal tender at point of sale. There are a few notable exceptions to this. Overstock.com, Inc. accepts, takes possession of, and holds cryptocurrency. It is illustrative to look at a recent 10-Q (Overstock.com, Inc., 2018). The first thing that is very interesting is the way the company describes itself in the cryptocurrency space. Overstock's overview is: "We are an online retailer and advancer of block chain technology". In their Accounting Policies section on page 10, Overstock lists cryptocurrency with prepaid and other current assets and states that gains and losses realized upon sale of cryptocurrencies are recorded in general and administrative expense. Overstock treats cryptocurrency as an intangible asset.

Another useful company to look at is Square, Inc. Square's core business is as a payment service for small businesses. According to a recent 10-Q (Square, Inc., 2018), Square allows businesses to accept cryptocurrency at point of sale. What is of interest is that Square holds cryptocurrency. On page 9 of its 10-Q, Square describes its accounting policy for cryptocurrency:

"The Company purchases bitcoin from private broker dealers or from customers. Upon purchase, the Company records the cost of bitcoin within other current assets in its consolidated balance sheets. Upon sale, the Company records the total sale amount received from customers as bitcoin revenue and the associated cost as cost of revenue." We can see Square also treats cryptocurrency as an intangible asset.

\section{Risks of Accepting Cryptocurrency}

Square, Inc. referenced above has identified various risks of accepting and holding cryptocurrency. Their 10-K section "Risk Factors" details specific risks associated with Bitcoin (Square, Inc., 2018). A key risk of holding Bitcoin is the possession of an online digital wallet. Square focuses on measures to protect this wallet from hackers. Square also lists more general risks that arise from Bitcoin not being legal tender or backed by any government.

More generally, a number of risks have received media attention and inquiry by regulatory agencies several instances follow:

- May 2018 the Justice Department launched a criminal investigation of potential market manipulation following large price swings into the value of Bitcoin. Investigators are looking into spoofing where traders submit false transactions and wash trades where traders buy and sell the same cryptocurrency giving a false view of market activity.

- March 2018 the IRS served Coinbase with a "John Doe" summon seeking information on their cryptocurrency transactions. The IRS is looking into whether Coinbase may have failed to pay federal taxes on their virtual currency transactions.

- July 2017 the SEC investigated whether DAO, an unincorporated organization, and Slock.it, a German corporation violated securities laws. According the SEC's Release No 81207 stated that "after DAO tokens were, sold, but before the DAO was able to commence funding projects, an attacker used a flaw in the DOA's code to steal approximately one-third of the DAO's assets". The investigation questioned whether the application of U.S federal securities laws were violated. The SEC issued a report on the investigation but did not pursue an enforcement action.

\section{Regulatory Environment}

\subsection{U.S. GAAP Guidance}

Accounting standards currently do not provide specific guidance on accounting for cryptocurrencies and so a holding entity should look to the nature of the cryptocurrency and their intended use in determining the most appropriate accounting treatment (EY, 2018).

Cryptocurrencies are often denominated in coins of tokens, often referred to as digital tokens, which come in different forms but can generally be classified into three broad categories (Currie, 2018).

- Digital Mediums of Exchange act like a digital wallet.

- Security Tokens which provide holders with voting rights or rights to participate in earnings and profits similar to a common stock security

- Utility Tokens which provide a right to obtain a current product or service from the issuing entity.

The form or nature of the digital token is defined by the issuing entity most often in the issuer's Initial Coin Offering 
(ICO) white paper.

\subsection{Holder's Intent}

When an entity determines that it owns the digital token it may plan to either convert immediately upon receipt into an accepted legal tender e.g. US Dollars or Euros or hold the token as an investment. The following describes possible accounting treatment for the three broad categories of digit tokens based upon the holder's intended use.

\subsubsection{Accounting for Digital Mediums of Exchange Tokens Where Intent is Immediate Exchange}

In the case of immediate conversion, the accounting is relatively straight forward. Using the current exchange rate of the entity's functional currency (e.g. US Dollar) the digital token is converted to cash and any gain or loss on the exchange is accounted for in other income or expense. When the holding entity intends to use the digital token as a medium of exchange ASC 350 Accounting for Intangible Assets would most likely apply, see 6.2.2 below.

\subsubsection{Accounting for Security Tokens Held for Investment (Currie, 2018)}

When a company's intention is to hold the digital token as an investment the accounting treatment gets murky. We examined current accounting standards to determine the most appropriate accounting treatment.

- $\quad$ Cash and Cash Equivalents: ASC 305 defines cash as currency, demand deposits with financial institutions and other accounts that have general characteristics of demand deposits Although digital tokens are sometimes referred to as a currency they are not legal tender and therefore do not qualify as cash or an equivalent under GAAP.

- $\quad$ Financial Instruments: ASC 825 defines a financial instrument as either cash or evidence of ownership in an entity through contractual arrangement. Security tokens may fall under this definition but do not generally provide a contractual right to receive cash or exchange for another recognized financial instrument.

However, a case can be made to account for security tokens as an equity security where the holder intends to hold the token as an investment. In this instance ASC 825 "Recognition and Measurement of Financial Assets and Financial Liabilities" requires the investment be measured at fair value with changes in fair value recognized in other net income or loss.

- Inventory: ASC 330 defines inventory as the aggregate of those items of tangible personal property that have the following characteristics:

- Held for sale in the ordinary course of business

- In process of production of such sales

- To be currently consumed in the production of good or services to be available for sale.

Some digital tokens are purchased or acquired with the intent to sell but do not qualify as tangible personal property under the definition of inventory per ASC 330.

- Intangible Asset: GAAP defines an intangible asset as those that lack physical substance. Security tokens and other types of cryptocurrencies might best fall within the category. ASC 350 "Intangibles-Goodwill and Other" provides some guidance requiring the investment to be recorded at cost unless impaired in which the asset is written down to fair value and the loss accounted for in other income/(loss). However, the nature of security tokens, their volatility, and the fact they may be traded on various exchanges makes these assets different from most intangible assets. Accordingly accounting for digital tokens as intangible assets may be conservative but perhaps not the most appropriate approach. Several of the "Big 4" accounting firm's suggest cryptocurrencies or digital tokens be accounted for as intangible assets in their technical or point of view publications and as noted earlier companies such as Overstock.com follow this accounting practice.

\subsubsection{Accounting for Utility Tokens}

These tokens provide the holder with a right to obtain product or service from the issuer and have some characteristics of intangible assets. Accordingly, under ASC 350 Intangibles-Goodwill and Other Assets and carried at cost (the value at the date of receipt) unless permanently impaired in which they would be written down to fair value and the loss recognized in other income/ (loss).

\subsection{SEC Regulations for the Issuances of Cryptocurrencies}

The SEC has not issued any specific regulations regarding accounting for cryptocurrencies. However, the Commission has stated that current securities law apply to the issuance of cryptocurrency, commonly referred to an 
Initial Coin Offering (ICO's). Section 2(a) (10) of the Securities Act and section 3(a) (10) of the Exchange Act provides that "a security includes an investment contract is an investment of money in a common enterprise or managerial efforts of another". The SEC issued release number 81207 "Report of Investigation Pursuant to Section 21(a) of the Securities Exchange Act of 1934 on July 25, 2017. This release provides detail on a specific cryptocurrency issuance and the SEC's position that an investment contract exists and that the investment of money need not take the form of cash. Release number 81207 goes onto to state that those who offer and sell securities in the United States must comply with securities law.

Based on our reading of the Securities and Exchange Acts and release number 81207 the SEC considers cryptocurrencies (or digital tokens) to be securities.

6.4 SEC Guidance Regarding Financial Statement Disclosures for Companies Accepting Cryptocurrency as Payment for Goods and Services?

As the SEC has not issued any specific guidance on financial statement disclosure for companies accepting cryptocurrency the existing rules regarding the application of GAAP in both the financial statements, footnotes or in the MD\&A section of SEC filings would continue to apply.

\subsection{SEC Framework for Cryptocurrency Companies to sell digital currencies}

In April 2019 the SEC issued the Framework for "Investment Contract" Analysis of Digital Assets. The framework discusses the offer, sale or distribution of a digital assets (sometimes referred to as an Initial Coin Offering or ICO) and whether U.S. Federal Securities laws would apply. Specifically, does a digital asset have the characteristics of one particular type of security - an Investment contract.

The framework provides that the issuer will need to analyze relevant transactions in order to determine if Federal Securities Law will apply. These criteria include the investment of money and reasonable expectation of profits.

The framework represents the views of the SEC but does not represent rules or regulations and a detail review of the framework should be completed before any determination is made.

\subsection{Internal Revenue Service}

The IRS position on cryptocurrencies is best described in Notice 2014-21. This notice describes how general tax principles apply to transactions using virtual currency, the IRS term for cryptocurrency and digital tokens. Notice 2014-21 stated "the IRS is aware that virtual currency maybe used to pay for goods or services or maybe held as an investment". Virtual currencies may operate like a real currency and maybe accepted as a medium of exchange, but do not have legal tender status in the opinion of the IRS. The Notice specifically refers to Bitcoin as an example of a convertible virtual currency and has an equivalent value in real currency.

Notice 2014-21 addresses tax treatment in a series of sixteen frequently asked questions (FAQ's). Our summary of the key FAQ's follows:

- Virtual currencies are treated as property and general tax principles apply. A tax payer who receives virtual currency as payment for goods or services must include in gross income the fair market value of the virtual currency as of the date received and the basis of the virtual currency is the U.S. as of the date of receipt.

- Virtual currencies, however, are not treated as currency that could generate foreign a currency gain or loss.

- A tax payer may have a gain or loss upon exchange of the virtual currency for other property if the fair market value exceeds or is less than its basis.

- The types of gains or losses realized on the sale or exchange of the virtual currency depends upon whether the virtual currency is a capital asset in the hands of the taxpayer.

- Lastly, payments in virtual currency to employees or independent contractors are to be included in the gross income or wages as remuneration for services, at fair market value, and are subject to withholding taxes by the employer.

\section{Discussion}

This paper presents a high-level view of cryptocurrency, its current use in business, and a summary of the regulatory environment. The topic is complex and evolving. Recently, Facebook, the world's largest social media company, released the white paper: An Introduction to Libra (Libra Association Members, 2019). This paper outlines Facebook's proposed cryptocurrency, blockchain, and governance. On this news, the US House of Representatives Committee on Financial Services held the hearing: Examining Facebook's Proposed Cryptocurrency and Its Impact 
on Consumers, Investors, and the American Financial System (2019). The hearing in its entirety is worth reading to anyone with an interest in cryptocurrency and blockchain. During the hearing, Gary Gensler provided testimony that "Facebook's ambitious proposal to enter the payment space with a digital token, Libra, raises many significant public policy considerations. These range from investor protection to privacy, systemic risk, guarding against illicit activity, monetary policy, government debt management, tax compliance and consumer protection." With a company as large as Facebook entering the cryptocurrency space, we expect continued enhancements in daily applications of cryptocurrency and blockchain along with increased regulation.

\section{Acknowledgements}

The authors would like to thank the anonymous reviewers for their valuable feedback.

\section{References}

Bitpay. (2018). Wallet Terms of Use. Retrieved from https://bitpay.com/about/terms\#fees-and-settlement

Bradley, R. (2018). Blockchain explained in under 100 words. Retrieved from https://www2.deloitte.com/ch/en/pages/strategy-operations/articles/blockchain-explained.html

Buterin, V. (2014, July 29). Vitalik Buterin explains Ethereum [Video file]. Retrieved August 19, 2018, from https://www.youtube.com/watch?v=TDGq4aeevgY

Currie, C. (2018). Cryptocurrencies: Time to consider plan B. Retrieved from https://www.pwc.com/us/en/cfodirect/publications/point-of-view/cryptocurrency-bitcoin-accounting.html

Coinbase. (2018) What happens if a merchant payment window expires. Retrieved from https://support.coinbase.com/customer/en/portal/articles/1544747-what-happens-if-a-merchant-payment-windo w-expires-

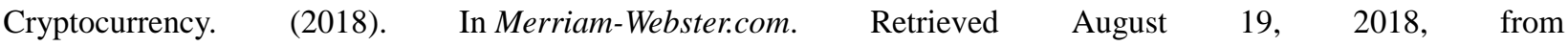
https://www.merriam-webster.com/dictionary/cryptocurrency

Examining Facebook's Proposed Cryptocurrency and Its Impact on Consumers, Investors, and the American Financial System: Hearings before the Committee on Financial Services, House, $116^{\text {th }}$ Cong. 1 (2019)

EY. (2018). Technical Line, A Holders's Accounting for Cryptocurrencies. Retrieved from

https://www.ey.com/publication/vwluassetsdld/technicalline_04623-181us_cryptocurrency_18october2018/\$file/tech nicalline_04623-181us_cryptocurrency_18october2018.pdf?OpenElement

FASB (Financial Accounting Standards Board). (2011, September 8). Accounting Standards Update (ASU) No. 2011-08. Accounting standards codification. Retrieved from http://asc.fasb.org/

FASB (Financial Accounting Standards Board). (2016, January 1). Accounting Standards Update (ASU) No. 2016-01. Accounting standards codification. Retrieved from https://asc.fasb.org/imageRoot/33/77884633.pdf

Fortney, L. (2019). Blockchain Explained. Retrieved from https://www.investopedia.com/terms/b/blockchain.asp

Libra Association Members. (2019). An Introduction to Libra., [online] Available: https://libra.org/en-US/white-paper/

Nakamoto, S. (2008). Bitcoin: A Peer-to-Peer Electronic Cash System., [online] Available: https://bitcoin.org/bitcoin.pdf.

Overstock.com, Inc. (2018). $2017 \quad 10-\mathrm{K} \quad$ Form. $\quad$ Retrieved from https://www.sec.gov/Archives/edgar/data/1130713/000113071318000019/ostk-20171231x10k.htm

Square, $\quad$ Inc. $\quad$ (2018). $2017 \quad 10-\mathrm{K} \quad$ Form. from https://www.sec.gov/Archives/edgar/data/1512673/000151267318000004/a10-kfilingsquareinc2017.htm

The Securities and Exchange Commission (SEC). (2017) Report of Investigation 34-81207 date July 25, 2017. Report of Investigation Pursuant to Section 21 (a) of the Securities Exchange Act of 1934. The DAO. Retrieved from https://www.sec.gov/litigation/investreport/34-81207.pdf 